\title{
Dynamical Jahn-Teller effects on the generation of electronic ring currents by circularly polarized light
}

\author{
Krishna R. Nandipati $\odot^{1, *}$ and Oriol Vendrell $\odot^{1,2, \dagger}$ \\ ${ }^{1}$ Theoretische Chemie, Physikalisch-Chemisches Institut, Universität Heidelberg, Im Neuenheimer Feld 229, 69120 Heidelberg, Germany \\ ${ }^{2}$ Centre for Advanced Materials, Universität Heidelberg, Im Neuenheimer Feld 205, 69120 Heidelberg, Germany
}

(Received 26 May 2021; accepted 9 September 2021; published 8 October 2021; corrected 12 November 2021)

\begin{abstract}
The generation of electronic ring currents in ring-shaped molecules by photoexcitation with circularly polarized laser light is considered in the presence of vibronic coupling effects. $(E \times e)$ Jahn-Teller distortions, unavoidable by symmetry in the $(E)$ subset of electronic states supporting the ring current, mix the clockwise and anticlockwise circulation directions of the electrons and can suppress the maximum achievable current by at least one order of magnitude, already for moderate vibronic coupling strengths, as compared to the Born-Oppenheimer limit of fixed atomic positions. The circulation direction of the electrons is found to depend on the spectral region of the $(E \times e)$ Hamiltonian. This fact results in the surprising effect that the same polarization direction of the laser pulse can trigger either clockwise or anticlockwise electronic dynamics depending on the wavelength of the photons. These findings are illustrated in a model of the triazine molecule.
\end{abstract}

DOI: 10.1103/PhysRevResearch.3.L042003

There is a growing interest in inducing, probing, and controlling electronic ring currents in ring-shaped molecular systems due to their potential applications in the next generation of optoelectronic devices [1-10]. Recent experiments realized ring currents in ring-shaped organic chromophores with strong magnetic fields and showed that the light absorption properties of the molecules were substantially modified [1]. From a theory perspective, it is well understood that circularly polarized light can also trigger ring currents by resonantly exciting the electrons to a manifold of doubly degenerate electronic states of $(E)$ symmetry related to a symmetry axis of the molecule [3,11-15]. It is inevitable by symmetry, though, that the $(E \times e)$ Jahn-Teller (JT) effect couples the $(E)$ electronic states and $(e)$ vibrational modes in all molecular point groups able to support ring currents [16-19], thus potentially mixing the two pristine ring-current circulation directions defined in the uncoupled limit [20,21]. The effects of the vibrational-electronic (vibronic) coupling in $(E \times e)$ JT (and Renner-Teller) systems are well understood in the areas of electronic spectroscopy [22-24], ultrafast vibronic dynamics [25,26], and charge migration [27]. However, it is not yet clear how vibronic couplings effects, particularly the dynamical $(E \times e)$ JT effect [17], modify the properties of the ring currents, limit their magnitude and stability, and thus their controllability.

\footnotetext{
*krishna.nandipati@pci.uni-heidelberg.de

†oriol.vendrell@uni-heidelberg.de

Published by the American Physical Society under the terms of the Creative Commons Attribution 4.0 International license. Further distribution of this work must maintain attribution to the author(s) and the published article's title, journal citation, and DOI.
}

In this Letter, we describe the theory of photoinduced molecular ring currents under nuclear-electronic couplings. We then apply this theory to the general $(E \times e)$ JT Hamiltonian, where the vibronic coupling results in a conical intersection $(\mathrm{CI})$ between the corresponding adiabatic potential energy surfaces and to a complex vibronic spectrum at moderate to strong couplings [17]. The $(E \times e)$ JT Hamiltonian constitutes the basis on which the description of more complex vibronic interactions, involving multimode effects in polyatomic molecules, have been built [19]. Its general features have been well known since the pioneering works of Longuet-Higgins and others [17,18,28,29], making it an ideal model to unravel the connection between molecular ring currents and nonadiabatic effects in molecules. The dramatic effects of the vibronic coupling on the photoinduced ring currents are finally demonstrated on the JT Hamiltonian of the sym-triazine molecule [30].

Within a diabatic representation of electronic states $\left|\Phi_{l}\left(\boldsymbol{Q}_{0}\right)\right\rangle$ defined at the reference nuclear configuration $\mathbf{Q}_{0}$ [19], the $j$ th eigenstate of the total molecular Hamiltonian can be written as

$$
|j\rangle=\sum_{l}\left|\Xi_{l}^{(j)}\right\rangle \otimes\left|\Phi_{l}\left(\boldsymbol{Q}_{0}\right)\right\rangle,
$$

where $\left|\Xi_{l}^{(j)}\right\rangle$ indicates the nuclear wave function corresponding to the $l$ th electronic state. Here and in the following, boldfaced quantities $\boldsymbol{q}$ and $\boldsymbol{Q}$ denote the collective coordinates of electrons and nuclei, respectively, and the $\vec{q}_{r}=(x, y, z)_{r}$ indicate the spatial coordinates of the $r$ th electron.

We consider now the doubly degenerate $(E)$ subspace of excited electronic states $\left[\left|\Phi_{x}\left(\boldsymbol{Q}_{0}\right)\right\rangle,\left|\Phi_{y}\left(\boldsymbol{Q}_{0}\right)\right\rangle\right]$ defined at the minimum energy geometry $\boldsymbol{Q}_{0}$ of the totally symmetric ground electronic state $\left|\Phi_{A}\left(\boldsymbol{Q}_{0}\right)\right\rangle$. Doubly degenerate subspaces are always present in molecules with a rotation axis 
$C_{n}$ of order $n \geqslant 3$, which enable degenerate electronic ring currents in the two rotation directions around this symmetry element $[3,11,31]$. To define these currents, one needs to introduce the complex linear combination of the two real orthonormal electronic configurations

$$
\left|\Phi_{ \pm}\left(\boldsymbol{Q}_{\mathbf{0}}\right)\right\rangle=\frac{1}{\sqrt{2}}\left[\left|\Phi_{x}\left(\boldsymbol{Q}_{\mathbf{0}}\right)\right\rangle \pm i\left|\Phi_{y}\left(\boldsymbol{Q}_{\mathbf{0}}\right)\right\rangle\right] .
$$

The vibronic coupling [cf. Eq. (7)] mixes the two (E) electronic configurations and the vibronic eigenstates $|j\rangle$ of the total molecular Hamiltonian can thus each be written as

$$
|j\rangle=\left|\Xi_{+}^{(j)}\right\rangle \otimes\left|\Phi_{+}\left(\boldsymbol{Q}_{0}\right)\right\rangle+\left|\Xi_{-}^{(j)}\right\rangle \otimes\left|\Phi_{-}\left(\boldsymbol{Q}_{0}\right)\right\rangle .
$$

In the above expression, we assume that the vibronic coupling mechanism only mixes the two components of the $(E)$ states with each other, which is the case in the $(E \times e)$ JT Hamiltonian. In general though, the $(E)$ states may also couple to states of other symmetry representations, for example $(A)$ and/or $(B)$, in particular point groups $[19,22,23]$. These other electronic states do not contribute to the ring current and we can neglect them for the purposes of our analysis. Starting with ansatz (3), and inserting the electronic current operator related to the electronic charge density via the continuity equation [32], one arrives at an expression for the one-body electronic current of the $j$ th vibronic eigenstate $\vec{J}_{j}(\vec{q})$ [cf. the Supplemental Material (SM) [33] for the complete derivation]. By introducing cylindrical coordinates $\vec{q}=(r, \theta, z)$ for the electrons, integrating $\vec{J}_{j}(\vec{q})$ over the radial and axial coordinates, and averaging the remaining flux over the $\theta$ angle, one arrives at [33]

$$
\mathcal{C}_{j}=\frac{\hbar}{m_{e}}\left(P_{+}^{(j)}-P_{-}^{(j)}\right)
$$

for the ring current of the vibronic eigenstate $|j\rangle$ of the full vibrational-electronic Hamiltonian, where $P_{ \pm}^{(j)}=\left\langle\Xi_{ \pm}^{(j)} \mid \Xi_{ \pm}^{(j)}\right\rangle$. This state-specific current is proportional to the imbalance of the population of the $E_{+}$and $E_{-}$electronic states in $|j\rangle$. If one considers the time-dependent Born-Huang expansion [34]

$$
|j(t)\rangle=\left|\Xi_{+}(t)\right\rangle \otimes\left|\Phi_{+}\left(\boldsymbol{Q}_{0}\right)\right\rangle+\left|\Xi_{-}(t)\right\rangle \otimes\left|\Phi_{-}\left(\boldsymbol{Q}_{0}\right)\right\rangle
$$

instead of the eigenstates (3), the same train of arguments follows and the time-dependent current of the wave packet reads [33]

$$
\mathcal{C}(t)=\frac{\hbar}{m_{e}}\left[P_{+}(t)-P_{-}(t)\right] .
$$

Equations (4) and (6) are our main working equations.

All molecular point groups with at least one rotational axis of order $n \geqslant 3$ have one $(E)$ representation that transforms as the $(x, y)$ components of the dipole operator, where $(x, y)$ is the plane of rotation perpendicular to the axis [18,35]. These are, for example, the $\left(E_{1 u}\right)$ and $\left(E_{u}\right)$ representations in the $D_{6 h}$ and $D_{4 h}$ point groups of benzene [15] and porphyrins [31], respectively. Therefore, one-photon electronic transitions from the totally symmetric ground-electronic state to the $(E)$ states are allowed and the interaction of the molecules with circularly polarized light in the molecular $(x, y)$ plane can generate ring currents owing to angular momentum conservation in the photoabsorption process $[20,36]$.
The interaction between the molecule and light propagating along the $z$ direction is described in the electric dipole approximation and the electromagnetic radiation is treated classically. The interaction term thus reads $-\left[\hat{\mu}_{x} \mathcal{E}_{x}(t)+\hat{\mu}_{y} \mathcal{E}_{y}(t)\right]$, with transition dipole operators $\hat{\mu}_{v}=$ $\mu_{A E}\left[\left|\Phi_{v}\left(\boldsymbol{Q}_{0}\right)\right\rangle\left\langle\Phi_{A}\left(\boldsymbol{Q}_{0}\right)\right|+\right.$ H.c. $]$. The electric field $\mathcal{E}_{v}(t)$ is derived from the vector potential $\mathcal{A}_{v}(t)=\frac{\mathcal{E}_{0}}{\omega_{L}} S(t) \sin \left(\omega_{L} t-\phi_{v}\right)$ as $\mathcal{E}_{v}(t)=-\partial A_{v}(t) / \partial t$. The pulse envelope is taken to be $S(t)=\tilde{\Theta}(t-\tau) \sin ^{2}\left(\frac{\pi t}{\tau}\right)$, and $\mathcal{E}_{0}, \tau$, and $\omega_{L}$ are the maximum amplitude, pulse duration (start to end), and carrier frequency of the pulse, respectively, and $\tilde{\Theta}(t-\tau)$ is the inverse Heaviside step function. For circular polarization, all pulse parameters for both $v \rightarrow(x, y)$ polarization directions are taken to be equal except for the $\phi_{v}$ phases. These are $\left(\phi_{x}=0, \phi_{y}=\right.$ $\pi / 2$ ) for a left circularly polarized pulse (LCP) and ( $\phi_{x}=$ $\pi / 2, \phi_{y}=0$ ) for a right circularly polarized pulse (RCP).

We apply the above considerations to the $(E \times e)$ JT model system with the Hamiltonian

$$
\begin{aligned}
\hat{H} & =\hat{T}_{N}+\hat{H}_{\mathrm{el}} \\
& =\hat{T}_{N}+\frac{\omega}{2} \rho^{2} \boldsymbol{I}_{3 \times 3}+\left(\begin{array}{ccc}
\epsilon_{+} & 0 & \kappa \rho e^{-i \alpha} \\
0 & \epsilon_{A} & 0 \\
\kappa \rho e^{i \alpha} & 0 & \epsilon_{-}
\end{array}\right),
\end{aligned}
$$

written here in its polar representation for the $(e)$ vibrational modes $(\rho, \alpha)[17,19]$. The electronic part of the total molecular Hamiltonian $\hat{H}_{\mathrm{el}}$ is represented in the diabatic basis $\left\{\left|\Phi_{A}\left(\boldsymbol{Q}_{0}\right)\right\rangle,\left|\Phi_{ \pm}\left(\boldsymbol{Q}_{0}\right)\right\rangle\right\}$ and includes an off-diagonal first-order JT interaction between the $(E)$ electronic states and $(e)$ vibrational modes [19]. $\boldsymbol{I}_{3 \times 3}$ is the $3 \times 3$ unit matrix, and $\omega, \epsilon_{ \pm, A}$, and $\kappa$ are the frequency of the $(e)$ modes, the energy of the diabatic electronic states at the reference geometry $\boldsymbol{Q}_{0}$ (i.e., $\rho=0$ ), and the linear JT coupling parameter, respectively. The vibronic interaction between the two $(E)$ states can be characterized by the dimensionless coupling strength parameter $\kappa / \omega$. The diagonalization of the $H_{\mathrm{el}}$ matrix as a function of the $(\rho, \alpha)$ coordinates yields the adiabatic potential energy surfaces with the "Mexican-hat" shape meeting at a CI at $\rho=0$ [37].

The effect of the vibronic coupling on the ring currents is most conveniently described by introducing the eigenstates of the two-dimensional (2D) harmonic oscillator (HO) in the polar representation $\chi_{n, m}(\rho, \alpha)=\langle\rho, \alpha \mid n, m\rangle$, where $n$ and $m$ are the radial and angular momentum quantum numbers of the 2D HO [38]. These can be extended to $|n, m, l\rangle$, including the $l$ quantum number for the electronic angular momentum operator around the rotational axis and defined by $\hat{L}_{\mathrm{el}}\left|\Phi_{ \pm}\left(\boldsymbol{Q}_{0}\right)\right\rangle= \pm \hbar\left|\Phi_{ \pm}\left(\boldsymbol{Q}_{0}\right)\right\rangle$ and $\hat{L}_{\mathrm{el}}\left|\Phi_{A}\left(\boldsymbol{Q}_{0}\right)\right\rangle=0$. The total angular momentum operator in the $(E \times e) \mathrm{JT}$ system thus reads $-i \hbar 2 \partial / \partial \alpha+\hat{L}_{\mathrm{el}}$ (cf. Ref. [17] for a discussion on the factor 2 for the vibrational term) and a ring current operator $\hat{\mathcal{C}}=\hat{L}_{\mathrm{el}} / m_{e}$ can be introduced by comparing Eqs. (4) and (6) with the definition of $\hat{L}_{\mathrm{el}}$. Now, the vibronic eigenstates of the total Hamiltonian can be classified by the vibronic angular momentum quantum number $q=2 m+l$, which is conserved under the vibronic coupling [17]. The totally symmetric $\left|\Phi_{A}\left(\boldsymbol{Q}_{0}\right)\right\rangle$ electronic state participates only in the $q=0$ block, whereas the $\left|\Phi_{ \pm}\left(\boldsymbol{Q}_{0}\right)\right\rangle$ states span both $q= \pm 1$ blocks 


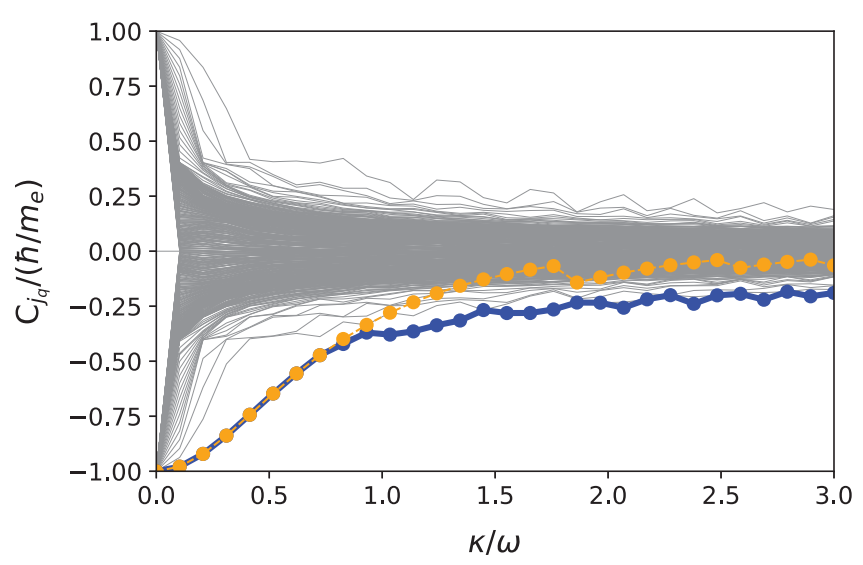

FIG. 1. Ring current $C_{j_{q}}$ values in $\hbar / m_{e}$ units for the eigenstates of the $q=-1$ block as a function of the coupling strength $\kappa / \omega$. The dotted-dashed curve in orange represents the current of the most optically bright eigenstate while the blue curve indicates the largest ring current achievable at a specific coupling strength.

due to the vibronic coupling. The JT coupling matrix elements of $\hat{H}$ in the $|n, m, l\rangle$ basis are given in the SM for completeness $[33,39]$.

Dipolar transitions from the ground electronic state fulfill the selection rule $\Delta q= \pm 1$ and circularly polarized radiation can only induce transitions $(0 \leftrightarrow 1)$ for LCP and $(0 \leftrightarrow-1)$ for RCP [20]. Finally, the vibronic eigenstates $\left|j_{q}\right\rangle$ of the complete Hamiltonian (7) in the $q=1$ block are one-to-one degenerate with the eigenstates of the $q=-1$ block. The degenerate pairs are related by the reversal of the signs of the $l$ and $m$ quantum numbers in all basis states $|n, m, l\rangle$ in their basis set expansion [17,30]. As a result, the electronic populations and currents of the vibronic eigenstates fulfill $P_{ \pm}^{\left(j_{ \pm 1}\right)}=P_{\mp}^{\left(j_{\mp 1}\right)}$ and $\mathcal{C}_{j_{+1}}=-\mathcal{C}_{j_{-1}}$ [cf. Eq. (4) and SM [33]].

In the following discussion we fix $\omega=0.003$ a.u. $\left(\approx 660 \mathrm{~cm}^{-1}\right)$, and $\epsilon_{ \pm}-\epsilon_{A}=7 \mathrm{eV}$, both in the typical range for molecular vibrations and vertical electronic transitions in molecules as, e.g., sym-triazine $\left(D_{3 h}\right)$ [30] and benzene $\left(D_{6 h}\right)$ [40]. The magnitude of the ring current $\left\langle j_{q}|\hat{\mathcal{C}}| j_{q}\right\rangle m_{e} / \hbar$ (in dimensionless units) is strongly affected by the vibronic coupling strength $\kappa / \omega$, as seen in Fig. 1 for the $q=-1$ block. In the weak vibronic coupling regime, i.e., $\kappa / \omega \ll 1$, the ring current supported by each eigenstate is close to either 1 or -1 . In the limit $\kappa \rightarrow 0,|0,0,-1\rangle$ coincides with the eigenstate with the largest transition dipole matrix element $\left|\left\langle 0,0,0|\hat{\mu}| j_{-1}\right\rangle\right|$, which is also the state that supports the largest ring current (in absolute terms). As the vibronic coupling increases towards $\kappa / \omega=1.0$, the state with the largest transition dipole does not necessarily coincide with the state featuring the largest current (cf. blue and orange curves in Fig. 1). The state-dependent ring current of all states quickly decreases as the coupling increases due to the progressive mixing of the left $\left(E_{-}\right)$and right $\left(E_{+}\right)$circulations of the electrons and the participation of high-lying vibrational levels. For stronger coupling, $\kappa / \omega>2$, the largest attainable current for a single eigenstate reaches an average $\sim 20 \%$ of the magnitude of the current achievable in the zero coupling limit (or by artificially fixing the molecular geometry to $\boldsymbol{Q}_{0}$ ).
The stationary ring current in a specific molecular eigenstate [cf. Eq. (4)] can be achieved by a long and narrowbandwidth circularly polarized pulse, whereas time-dependent ring currents [cf. Eq. (6)] can be obtained with a circularly polarized pulse of sufficient bandwidth that is resonant with a set of molecular eigenstates of the same $q$ block. While considering phototriggered ring currents, we set the product of the electric field amplitude and the transition dipole matrix element, $\mathcal{E}_{0} \cdot \mu_{A E}$, low enough to remain in the regime of first-order perturbations. In this regime, the ring currents are intensity independent when normalized by the amount of total excitation $P_{\mathrm{ex}}\left(t_{f}\right)=\left[1-P_{0}\left(t_{f}\right)\right]$, where $P_{0}(t)$ is the population of the absolute ground state and $t_{f}$ is the final time of the simulation after the pulse is over. $P_{\mathrm{ex}}\left(t_{f}\right)$ is kept in the order of $5 \times 10^{-2}$ or lower.

In the following we consider phototriggered ring currents in an $(E \times e) \mathrm{JT}$ system with a $\kappa / \omega \approx 2.2$, the value corresponding to the sym-triazine system [30]. We consider both long (250 fs) and short (30 fs) RCP pulses, whose spectrum is shown superimposed in Figs. 2(a) and 2(b) in red and green, respectively. At about $6.8 \mathrm{eV}$, the long pulse is resonant with the eigenstate featuring the largest transition dipole moment. This state is part of the lower-energy absorption band, which is energetically located below the CI (marked with a cross on the energy axis). The bandwidth of the pulse is narrow enough to overlap effectively with only one vibronic eigenstate, which results in a stationary ring current after the pulse is over, as indicated by the red curve in Fig. 2(c). As expected, the excitation-normalized stationary ring current coincides with the ring current of the corresponding eigenstate, $\mathcal{C}_{j_{q}}=\mathcal{C}\left(t_{f}\right) / P_{\mathrm{ex}}\left(t_{f}\right)$, about -0.075 in $\hbar / m_{e}$ units. The shorter pulse, in contrast, overlaps with several eigenstates, resulting in a coherent excitation and an oscillatory ring current [cf. the green curve in Fig. 2(c)]. Because the eigenstates in this spectral region are characterized by an clockwise electronic circulation (as seen from the laser source), as indicated by the negative $C_{j} /\left(\hbar / m_{e}\right)$ values shown in blue in Fig. 2(a), the coherent oscillatory current does not change its direction and its time-averaged value is negative.

As a comparison, we consider now the spectral region at about $7.2 \mathrm{eV}$, and thus above the $\mathrm{CI}$ as shown in Fig. 2(b). Specific eigenstates in this spectral region can be identified with either right or left circulation of the electrons. The long pulse is resonant with one single eigenstate and results, again, in a stationary ring current [cf. red curve in Fig. 2(d)], although of larger magnitude $(\sim 0.14)$ than before and, remarkably, with opposite circulation direction of the electrons as compared to the long pulse with a lower photon energy. Thus, different spectral regions of a JT system may feature opposite circulation directions of the electrons, which is a direct consequence of the vibronic coupling effects and of the mixing of the electronic and vibrational angular momenta. Therefore, a RCP does not necessarily result in clockwise circulation of the electrons, as in the $\kappa \rightarrow 0$ limit. A shorter pulse in this spectral region results in an oscillatory current [cf. the green curve in Fig. 2(d)] which, however, oscillates now with both positive and negative ring current values. The ring current changes direction periodically although it remains positive when time averaged. 

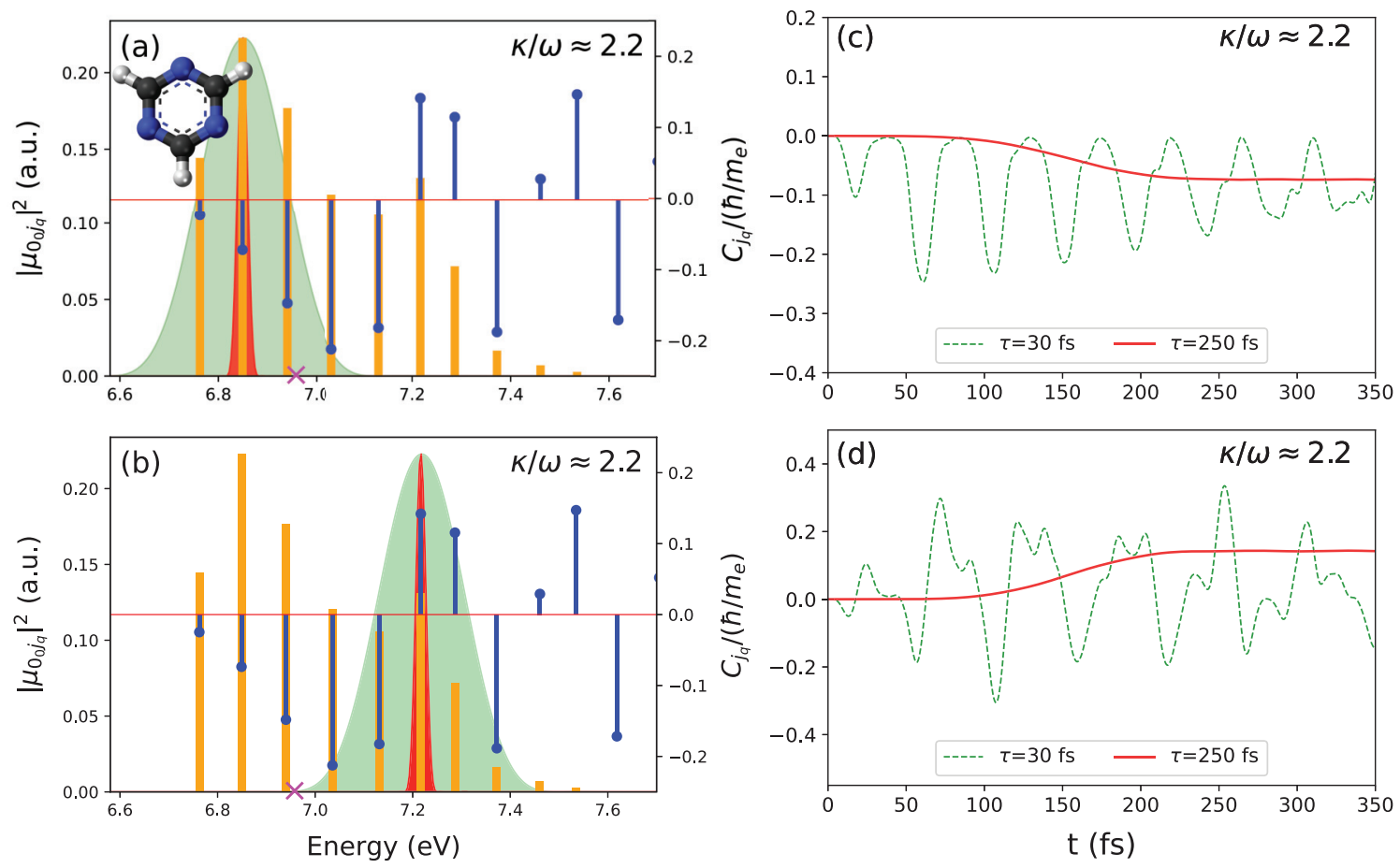

FIG. 2. (a) Absorption spectrum $\left(\propto\left|\mu_{0_{0} j_{q}}\right|^{2}\right)$ for the 2D sym-triazine $(E \times e)$ JT Hamiltonian (orange, left $y$ scale) and eigenstate ring currents $\left[\mathcal{C}_{j_{q}} /\left(\hbar / m_{e}\right)\right]$ (blue, middle $y$ scale) as a function of the eigenstates' energy superimposed with the spectral representation of the short ( $\tau=30 \mathrm{fs}$, shaded in green) and long ( $\tau=250 \mathrm{fs}$, shaded in red) RCPs resonant with the lower band of the absorption spectrum. (b) Same as (a) but pulses are resonant with the spectral region above the CI (marked $\times$ on the energy axis). (c), (d) Time-dependent ring currents $\left[\mathcal{C}(t) /\left(\hbar / m_{e}\right) / P_{\mathrm{ex}}\left(t_{f}\right)\right]$ generated by the pulses shown in (a) and (b), respectively. The time-dependent ring currents are normalized by the total excitation probability $P_{\mathrm{ex}}\left(t_{f}\right)$ after the pulse (cf. main text). The inset in (a) shows the geometry of sym-triazine and the horizontal red line in (a) and (b) is to guide the eye.

Finally, it is interesting to note that the energetic proximity of the $\mathrm{CI}$ at about $7 \mathrm{eV}$ has no particular consequences for the ring currents. The ground vibronic eigenstate $\left|0_{-1}\right\rangle$ of the $q=-1$ block is found to be at about $6.8 \mathrm{eV}$ with the current approaching zero. This state lies further deep in the "Mexican-hat" adiabatic potentials at the coupling $\kappa / \omega>2.5$ (not shown), where the mixing of both circulation directions is very strong and no currents can be generated in this spectral region. As the eigenstates' energy increases and approaches the $\mathrm{CI}$ energy, $\mathcal{C}_{j_{q}}$ and $\left|\mu_{0_{0} j_{q}}\right|^{2}$ keep varying smoothly and no special effects related to the CI are present. Nonetheless, even at relatively large vibronic couplings it is possible to identify spectral regions where either stationary or oscillatory ring currents can be generated. Due to the vibronic coupling, however, these currents are about one order of magnitude smaller than the currents that can be generated in the $\kappa \rightarrow 0$ limit [in which case $\mathcal{C} /\left(\hbar / m_{e}\right)$ is \pm 1$]$. We remark that these and the above claims are not affected by the inclusion of second-order JT coupling (cf. Fig. S1 in SM and its discussion).
In conclusion, molecular systems with the necessary symmetry in their ground electronic state support stationary and oscillatory ring currents upon photoexcitation to an (E)-representation manifold of electronic states by circularly polarized light, even in the presence of strong vibronic coupling effects. We have arrived at expressions for such currents in terms of vibronic eigenstates and of timedependent nuclear-electronic wave packets, and applied the theory to the paradigmatic $(E \times e)$ JT Hamiltonian. The observations made on JT systems are general and have consequences for laser-generated ring currents and their control in complex molecules, and indicate that vibronic coupling effects are central and must be considered in future applications.

K.N. acknowledges the collaborative research center "SFB 1249: N-Heteropolyzyklen als Funktionsmaterialen" of the German Research Foundation (DFG) for financial support. The authors thank Prof. Horst Köppel and Prof. Wolfgang Domcke for helpful discussions.
[1] B. Kudisch, M. Maiuri, L. Moretti, M. B. Oviedo, L. Wang, D. G. Oblinsky, R. K. Prudhomme, B. M. Wong, S. A. McGill, and G. D. Scholes, Ring currents modulate optoelectronic properties of aromatic chromophores at 25 T, Proc. Natl. Acad. Sci. USA 117, 11289 (2020).
[2] K. Kaiser, L. M. Scriven, F. Schulz, P. Gawel, L. Gross, and H. L. Anderson, An sp-hybridized molecular carbon allotrope, cyclo[18]carbon, Science 365, 1299 (2019).

[3] I. Barth, J. Manz, Y. Shigeta, and K. Yagi, Unidirectional electronic ring current driven by a few cycle circularly polarized 
laser pulse: Quantum model simulations for Mg-porphyrin, J. Am. Chem. Soc. 128, 7043 (2006).

[4] J. E. Anthony, Functionalized acenes and heteroacenes for organic electronics, Chem. Rev. 106, 5028 (2006).

[5] E. Räsänen, A. Castro, J. Werschnik, A. Rubio, and E. K. U. Gross, Optimal Control of Quantum Rings by Terahertz Laser Pulses, Phys. Rev. Lett. 98, 157404 (2007).

[6] M. Galperin, M. A. Ratner, A. Nitzan, and A. Troisi, Nuclear coupling and polarization in molecular transport junctions: Beyond tunneling to function, Science 319, 1056 (2008).

[7] D. Rai, O. Hod, and A. Nitzan, Circular currents in molecular wires, J. Phys. Chem. C 114, 20583 (2010).

[8] K. K. Saha, B. K. Nikolić, V. Meunier, W. Lu, and J. Bernholc, Quantum-Interference-Controlled Three-Terminal Molecular Transistors Based on a Single Ring-Shaped Molecule Connected to Graphene Nanoribbon Electrodes, Phys. Rev. Lett. 105, 236803 (2010).

[9] C. Ramírez and L. Medina-Amayo, Scattering matrix of arbitrary tight-binding Hamiltonians, Ann. Phys. 378, 303 (2017).

[10] M. D. Peeks, T. D. W. Claridge, and H. L. Anderson, Aromatic and antiaromatic ring currents in a molecular nanoring, Nature (London) 541, 200 (2017).

[11] I. Barth and J. Manz, Quantum switching of magnetic fields by circularly polarized re-optimized $\pi$ laser pulses: From oneelectron atomic ions to molecules, in Progress in Ultrafast Intense Laser Science VI (Springer, Berlin, 2010), pp. 21-44.

[12] K.-J. Yuan, C.-C. Shu, D. Dong, and A. D. Bandrauk, Attosecond dynamics of molecular electronic ring currents, J. Phys. Chem. Lett. 8, 2229 (2017).

[13] D. Jia, J. Manz, B. Paulus, V. Pohl, J. C. Tremblay, and Y. Yang, Quantum control of electronic fluxes during adiabatic attosecond charge migration in degenerate superposition states of benzene, Chem. Phys. 482, 146 (2017).

[14] M. Kanno, H. Kono, and Y. Fujimura, Laser-control of ultrafast $\pi$-electron ring currents in aromatic molecules: Roles of molecular symmetry and light polarization, Appl. Sci. 8, 2347 (2018).

[15] C. M. Liu, J. Manz, K. Ohmori, C. Sommer, N. Takei, J. C. Tremblay, and Y. Zhang, Attosecond Control of Restoration of Electronic Structure Symmetry, Phys. Rev. Lett. 121, 173201 (2018).

[16] H. A. Jahn and E. Teller, Stability of polyatomic molecules in degenerate electronic states - I-orbital degeneracy, Proc. R. Soc. London, Ser. A 161, 220 (1937).

[17] H. C. Longuet-Higgins, U. Öpik, M. H. L. Pryce, and R. A. Sack, Studies of the Jahn-Teller effect. II. The dynamical problem, Proc. R. Soc. London, Ser. A 244, 1 (1958).

[18] R. Englman, The Jahn-Teller Effect in Molecules and Crystals (Wiley, New York, 1972).

[19] H. Köppel, W. Domcke, and L. Cederbaum, Multimode molecular dynamics beyond the Born-Oppenheimer approximation, Adv. Chem. Phys. 57, 59 (1984).

[20] K. R. Nandipati and O. Vendrell, On the generation of electronic ring currents under vibronic coupling effects, J. Chem. Phys. 153, 224308 (2020).

[21] M. Kanno, H. Kono, Y. Fujimura, and S. H. Lin, Nonadiabatic Response Model of Laser-Induced Ultrafast $\pi$-Electron Rotations in Chiral Aromatic Molecules, Phys. Rev. Lett. 104, 108302 (2010).

[22] W. Domcke, D. Yarkony, and H. Köppel, Conical Intersections: Electronic Structure, Dynamics \&
Spectroscopy, Vol. 15 (World Scientific, Singapore, 2004).

[23] W. Domcke, D. R. Yarkony, and H. Köppel, Conical Intersections: Theory, Computation and Experiment, Vol. 17 (World Scientific, Singapore, 2011).

[24] C. Jungen, The Renner-Teller effect revisited 40 years later, J. Mol. Spectrosc. 363, 111172 (2019).

[25] Z. Li, M. E.-A. Madjet, O. Vendrell, and R. Santra, Correlated Dynamics of the Motion of Proton-Hole Wave Packets in a Photoionized Water Cluster, Phys. Rev. Lett. 110, 038302 (2013).

[26] C. Arnold, O. Vendrell, and R. Santra, Electronic decoherence following photoionization: Full quantum-dynamical treatment of the influence of nuclear motion, Phys. Rev. A 95, 033425 (2017).

[27] V. Despré, A. Marciniak, V. Loriot, M. C. E. Galbraith, A. Rouzée, M. J. J. Vrakking, F. Lépine, and A. I. Kuleff, Attosecond hole migration in benzene molecules surviving nuclear motion, J. Phys. Chem. Lett. 6, 426 (2015).

[28] W. Moffitt and A. Liehr, Configurational instability of degenerate electronic states, Phys. Rev. 106, 1195 (1957).

[29] M. D. Sturge, The Jahn-Teller effect in solids, Solid State Phys. 20, 91 (1968)

[30] R. L. Whetten, K. S. Haber, and E. R. Grant, The dynamic JahnTeller effect in sym-triazine: Nonadiabatic wave functions and hindered fluxionality, J. Chem. Phys. 84, 1270 (1986).

[31] I. Barth and J. Manz, Periodic electron circulation induced by circularly polarized laser pulses: Quantum model simulations for Mg porphyrin, Angew. Chem., Int. Ed. 45, 2962 (2006).

[32] A. Messiah, Quantum Mechanics (Dover, New York, 1999).

[33] See Supplemental Material at http://link.aps.org/supplemental/ 10.1103/PhysRevResearch.3.L042003 for the derivation of the ring current expression for the vibronically coupled $E$ states, coupling matrix elements of the $(E \times e) \mathrm{JT}$ Hamiltonian, and the eigenstates' ring current under quadratic $(E \times e) \mathrm{JT}$ coupling.

[34] M. Born and K. Huang, Dynamical Theory of Crystal Lattices (Clarendon, Oxford, UK, 1954).

[35] I. Barth, J. Manz, and L. Serrano-Andrés, Quantum simulations of toroidal electric ring currents and magnetic fields in linear molecules induced by circularly polarized laser pulses, Chem. Phys. 347, 263 (2008).

[36] S. Eckart, M. Kunitski, M. Richter, A. Hartung, J. Rist, F. Trinter, K. Fehre, N. Schlott, K. Henrichs, L. P. H. Schmidt et al., Ultrafast preparation and detection of ring currents in single atoms, Nat. Phys. 14, 701 (2018).

[37] I. B. Bersuker, The Jahn-Teller effect (Cambridge University Press, Cambridge, UK, 2006).

[38] L. Pauling and E. B. Wilson, Jr., Introduction to Quantum Mechanics: With Applications to Chemistry (McGraw-Hill, New York, 1935).

[39] G. Gallup, Matrix elements and eigenfunctions of multidimensional oscillators by operational methods: Part I. Two dimensional isotropic harmonic oscillator, J. Mol. Spectrosc. 3, 148 (1959).

[40] G. Worth, A model Hamiltonian to simulate the complex photochemistry of benzene, J. Photochem. Photobiol., A 190, 190 (2007).

Correction: A minor error in the caption to Figure 2 has been fixed. The acronym LCPs has been changed to RCPs. 